Research Paper

\title{
MiR-375 Has Contrasting Effects on Newcastle Disease Virus Growth Depending on the Target Gene
}

\author{
Xinglong Wang*, Yanqing Jia*, Xiangwei Wang, Chongyang Wang, Changie Lv, Xiaoqin Li, Zhili Chu, \\ Qingsong Han, Sa Xiao, Shuxia Zhang, Zengqi Yang \\ College of Veterinary Medicine, Northwest A\&F University, Yangling, Shaanxi Province 712100, PR China \\ * These authors contributed equally to this work. \\ $\bowtie$ Corresponding author: Email: yzq8162@163.com; Tel: +86-029-87091106 \\ (c) Ivyspring International Publisher. This is an open access article distributed under the terms of the Creative Commons Attribution (CC BY-NC) license \\ (https://creativecommons.org/licenses/by-nc/4.0/). See http://ivyspring.com/terms for full terms and conditions.
}

Received: 2018.01.23; Accepted: 2018.11.01; Published: 2019.01.06

\begin{abstract}
MicroRNAs regulate post-transcriptional gene expression via either translational repression or mRNA degradation. They have important roles in both viral infection and host anti-infection processes. We discovered that the miR-375 is significantly upregulated in Newcastle disease virus (NDV)-infected chicken embryonic visceral tissues using a small RNA sequencing approach. Further research revealed that the overexpression of miR-375 markedly decreases the replication of the velogenic NDV F48E9 and the lentogenic NDV La Sota by targeting the M gene of NDV in DF-1 cells. Interestingly, miR-375 has another target, ELAVL4, which regulates chicken fibrocyte cell cycle progression and decreases NDV proliferation. In addition, miR-375 can influence bystander cells by its secretion in culture medium. Our results indicated that miR-375 is an inhibitor of NDV, but can also enhance NDV growth by reducing the expression of its target ELAVL4. These results emphasize the complex roles of microRNAs in the regulation of viral infections.
\end{abstract}

Key words: Newcastle disease virus; microRNAs; miR-375; ELAVL4; M gene

\section{Introduction}

MicroRNAs (miRNAs) are a group of endogenous small non-coding RNAs of 21 to 25 nucleotides in length; they regulate the expression of target genes post-transcriptionally and have significant roles in various biological processes, including development, differentiation, proliferation, and apoptosis [1-3]. The majority of miRNAs are transcribed as part of annotated genes from independent transcription units located in the antisense orientation or the intergenic regions of annotated genes. In animals, miRNAs are transcribed as long primary transcripts (pri-miRNAs) and are further cropped by the nuclear RNase III Drosha into hairpin-shaped pre-miRNAs [4, 5]. These pre-miRNAs are subsequently cleaved by the cytoplasmic RNase III Dicer, after they are exported out of the nucleus by exportin-5 (Exp5), a member of the ran-dependent nuclear transport receptor family, into a $22-n t$ miRNA duplex [6-8]. One strand of the short-lived duplex remains as a mature miRNA, and another strand is degraded by an unknown nuclease. miRNA-induced silencing complexes (miRISCs) are formed following the loading of mature mRNA into Argonaute proteins (AGO2) [9]. Then, miRISCs bind to targets by base-pairing of the miRNA seed sequence with the mRNA target sites, leading to the accelerated degradation of mRNA or the blockade of translational processing bodies [10].

miRNAs have significant roles in viral infection, either in cellular antiviral responses or the replication and propagation of viruses, via complex regulatory pathways [11-14]. Several studies have shown that host miRNAs could promote or inhibit viral replication by targeting viral RNAs [15-18]. For example, miR-122, a liver-specific miRNA, could promote Hepatitis $C$ virus replication by binding to the $5^{\prime}$ UTR of the viral genome or by accelerating the ribosome and Ago 2 interactions with viral RNA [15, 
18-20]. In contrast, miR-296-5p suppresses Enterovirus 71 replication by targeting the coding regions of the viral capsid proteins VP1 and VP3 [16]. Many miRNAs, including MiR-342-5p, miR-485, and miR-548g-3p, are involved in antiviral processes by targeting the viral genome [17, 21, 22]. In addition, viral infections can also inhibit maturation or accelerate the degradation of host microRNAs involved in the antiviral response $[9,23]$.

Newcastle disease (ND) is one of the most economically important infectious diseases, causing high rates of death in chickens and birds and resulting in significant economic losses to the chicken industry worldwide each year [24-27]. Its causative pathogen is Newcastle disease virus (NDV), a small enveloped, linear, single negative-stranded RNA virus [28]. The genome of NDV encodes six structural proteins, NP, $\mathrm{P}, \mathrm{M}, \mathrm{F}, \mathrm{HN}$, and $\mathrm{L}[28,29]$. The $\mathrm{HN}$ and $\mathrm{F}$ proteins play important roles in viral invasion and membrane fusion, NP, P, and L constitute the RNA polymerase affecting viral replication, and the $\mathrm{M}$ protein is mainly involved in virion assembly and budding [29-31]. Although various vaccines have been used to control NDV, it is very difficult to eliminate or effectively control NDV infection using the current vaccination strategies owing to the high frequency of viral mutation, wide range of hosts, and the antibody-dependent enhancement of NDV [26, 27]. Therefore, it is imperative to study the mechanisms underlying the pathogenesis of NDV to develop more effective control measures.

Since miRNAs are involved in host-virus interactions, it is important to determine their roles in NDV infection. In fact, miR-485, targeting Retinoic acid-inducible gene I (RIG-I), plays a role in regulating NDV and Avian influenza virus H5N1 infections [17]. In the current study, the expression levels of miR-375 as well as several other miRNAs were up-regulated during NDV infection based on a next-generation sequencing analysis. MiR-375, in particular, effectively suppressed NDV replication and its functions were further investigated.

\section{Materials and Methods}

\section{Cells and viruses}

Chicken embryo fibroblast cells (DF-1), human laryngeal cancer epithelial cells (Hep2), and HEK293T cells were maintained in Dulbecco's modified Eagle's medium (DMEM; Gibco, Gaithersburg, MD, USA) supplemented with $10 \%$ fetal bovine serum (FBS; Hyclone, Logan, UT, USA). Ten-day-old SPF chicken embryos were obtained from Merial-vital Laboratory Animal Technology (Beijing, China) and maintained in an incubator at $37^{\circ} \mathrm{C}$.
The velogenic NDV strain F48E9 and lentogenic NDV strain rLa Sota-GFP (La Sota) were kept in our lab. Viruses were propagated in 9-day-old SPF chicken embryos, titrated by the hemagglutination assay (HA), and stored at $-80^{\circ} \mathrm{C}$ for later use.

\section{Virus infection and small RNA sequencing}

A total of $10^{4} \mathrm{PFU}$ of each virion of F48E9 or La Sota was independently injected into the allantoic cavity of 10-day-old chicken embryos. An equal volume of phosphate-buffered saline (PBS) was injected as a negative control. The visceral tissues of each group, including heart, liver, stomach, intestines and spleen, were collected at $36 \mathrm{~h}$ post-infection (hpi), and three small RNA sequencing samples were sent to Novogene for sequencing (Wuhan, China). RNA extraction, library preparation, and sequencing analyses were performed by Novogene. Raw reads were demultiplexed and trimmed to remove low-quality sequences prior to downstream analyses. To detect differentially expressed miRNAs, a corrected $p$-value (q-value) of $<0.01$ and | log2 (foldchange) $\mid$ of $>1$ were set as the threshold parameters for significance.

\section{Computational target prediction}

The miR-375 targets were predicted using the miRanda algorithm. The miR-375 binding sites in the ELAVL4 3' UTR were predicted using the TargetScan website (http://www.targetscan.org/mamm_31/). Predicted targets were selected according to the optimal complementarity between miRNAs and a given mRNA based on a computational TargetScan algorithm [32]. NDV genomes were predicted using BLAST.

\section{Plasmid construction and cell transfection}

The miR-375 expression plasmid p-miR-375 (miR-375), the miR-375 inhibitor plasmid p-sponge-375 (sponge-375), scramble control plasmid with mutation in binding site of miR-375 and negative control (miR-NC and sponge-NC) were purchased from GenePharma (Shanghai, China). The plasmids pluc- $\mathrm{M}_{\mathrm{F}}(\mathrm{FM})$ and pluc- $\mathrm{M}_{\mathrm{L}}(\mathrm{LM})$ were derived from the psiCHECK2 vector with an inserted $M$ gene from NDV strain F48E9 and La Sota, respectively. The full length of $\mathrm{M}$ gene of F48E9 and La Sota were respectively cloned into the pcDNA3.0 and pCAGGS vectors, and named as pcDNA3.0-F48E9 M (pcDNA3.0-FM) and pCAGGS-Flag-La Sota $\mathrm{M}$ (pCAGGS-Flag-LM), and the pcDNA3.0-FM has already been constructed and kept in our lab. The full length of chELAVL4 was cloned into the pCAGGS vector with a $\mathrm{HA}$ tag to obtain the plasmid pCAGGS-HA-ELAVL4. The shRNA primers targeting ELAVL4 were designed using the BLOCK-iT RNAi 
Designer website and the primer sequences are listed in Table 1. Next, double-stranded shRNA was synthesized and cloned into the plasmid pCD513B-U6-neo to obtain shRNA1, shRNA2, and negative control (NC). All primers used in this study are listed in Table 1, and all recombinant plasmids were verified by sequencing. Cell transfection was performed using the TurboFect Transfection Reagent (Thermo Scientific, Waltham, MA, USA) according to the manufacturer's instructions.

\section{Virus infection and cell treatment}

To validate miR-375 expression in NDV infected cells, DF-1 cells were seeded in 24-well plates for $24 \mathrm{~h}$, infected with F48E9 or La Sota (0.1 MOI), and collected at $6,12,18,24$, and 30 hpi to analyze miR-375 expression by RT-qPCR. After the transfection of DF-1 cells with miR-375, miR-NC, sponge-375, sponge-NC, pCAGGS-HA-ELAVL4, shRNA, or NC plasmids for $24 \mathrm{~h}$, the cells were infected with F48E9 or La Sota (0.1 MOI) and the supernatants were collected at 24, 36, and 48 hpi for viral titration.

NDV replication was investigated after DF-1 cells were treated with the supernatant containing miR-375, miR-NC, sponge-375, or sponge-NC for $24 \mathrm{~h}$ before infection with F48E9 or La Sota (0.1 MOI) or after incubation with F48E9 or La Sota (0.1 MOI) for 1 h. The supernatant was collected and viral titer changes were evaluated at $24 \mathrm{~h}$ after each treatment.

\section{Virus titration}

Viral progeny titers were quantified by $50 \%$ tissue culture infectious dose $\left(\mathrm{TCID}_{50}\right)$, fluorescence intensity, or plaque assays. Briefly, the DF-1 cells were seeded in 96-well or 24-well plates at $24 \mathrm{~h}$ before virus infection. The supernatant from cell cultures was 10-fold serially diluted and $100 \mu \mathrm{L} /$ well or 300 $\mu \mathrm{L} /$ well was added to the 96-well or 24 -well plates. At $5 \mathrm{~d}$ or $3 \mathrm{~d}$ post-infection, $\mathrm{TCID}_{50}$ was calculated using the Reed-Muench method [33], the average green fluorescent cell count was determined in each microscopic field as described previously [34], or the plaque forming units (PFU) were computed [35].

\section{Real-time quantitative PCR (RT-qPCR)}

The microRNAs were extracted using the miRcute miRNA Isolation Kit, and then cDNA was synthesized using the miRcute miRNA First-strand cDNA Synthesis Kit, as per the manufacturer's instructions (TIANGEN, Beijing, China). To quantify the viral load or viral mRNA, total RNAs were extracted using TRIzol Reagent, as per the manufacturer's instructions (Invitrogen, Carlsbad, CA, USA). Then, $2 \mu \mathrm{g}$ of total RNA from each sample was reverse transcribed into cDNA using the HiScript 1st Strand cDNA Synthesis Kit (Vazyme Biotech Co., Ltd., Nanjing, China) as per the manufacturer's instructions. Real-time quantitative PCR (RT-qPCR) was performed using a real-time thermocycler (Tianlong, Shaanxi, China) with SYBR green detection. Viral mRNA and miRNA abundances were measured by RT-qPCR and normalized against the level of $28 \mathrm{~S}$ mRNA.

\section{Immunoblotting}

The plasmids miR-375, miR-NC, sponge-375 or sponge-NC were co-transfected with pcDNA3.0-FM/pCAGGS-Flag-LM into DF-1 cells for $24 \mathrm{~h}$, and then, the cells were collected as reported previously [36] for detecting $M$ gene expression by immunoblotting.

Table 1. The primers used in this study

\begin{tabular}{|c|c|c|c|}
\hline Primers & Sequences (5'-3') & Size (bp) & $\mathrm{TM}\left({ }^{\circ} \mathrm{C}\right)$ \\
\hline pCAGGS-HA-ELAVL4-F & CCATCGATATGGAGTGGAATGGCCTGAAGATG & 1159 & 53 \\
\hline pCAGGS-HA-ELAVL4-R & CGGCTAGCTCAAGCGTAGTCTGGGACGTCGTATGGGTAGGATTTGTGGGTTTTATTGG & & \\
\hline pCAGGS-Flag-LM-F & GGAATTCATGGACTCATCTAGGACAAT & 1135 & 50 \\
\hline pCAGGS-Flag-LM-R & GAAGATCTTTACTTATCGTCGTCATCCTTGTAATCTTTCTTAAAAGGATTGT & & \\
\hline Luc-FM-F & CCGCTCGAGATGGACTCATCTAGGACAATTG & 567 & 52 \\
\hline Luc-FM-R & TTGCGGCCGCTTCGGCACCACAGTCAAAGAAAC & & \\
\hline Luc-LM-F & CCGCTCGAGATGGACTCATCTAGGACAATTG & 567 & 52 \\
\hline Luc-LM-R & TTGCGGCCGCTTCGGTACCACAGTCAAGGAGAC & & \\
\hline M-F & CCGATCGTCCTACAAGACACAG & 223 & 60 \\
\hline M-R & GGACGCTTCCTAGGCAGAGCAT & & \\
\hline NDV-F & ATGGGCYCCAGAYCTTCTAC & 535 & 55 \\
\hline NDV-R & CTGCCACTGCTAGTTGTGATAATCC & & \\
\hline ELAVL4-F & GGTATCGTATGCCCGTCCAA & 214 & 60 \\
\hline ELAVL4-R & GGCTTCTTCCGCCTCTATTC & & \\
\hline ELAVL4-shRNA1F & GATCCGGAATGGCCTGAAGATGATAA TTCAAGAGATTATCATCTTCAGGCCATTCCTTTTTTg & 63 & 37 \\
\hline ELAVL4-shRNA1R & AATTCAAAAAAGGAATGGCCTGAAGATGATAATTCAAGAGATTATCATCTTCAGGCCATTCCg & & \\
\hline ELAVL4-shRNA2F & GATCCGGAGTCTCTTTGGGAGCATTG TTCAAGAGACAATGCTCCCAAAGAGACTCCTTTTTTg & 63 & 37 \\
\hline ELAVL4-shRNA2R & AATTCAAAAAAGGAGTCTCTTTGGGAGCATTG TTCAAGAGACAATGCTCCCAAAGAGACTCCg & & \\
\hline shRNA-NC-F & GATCCGACCCGGCGACGGGCTAGTCTTCAAGAGAGACTAGCCCGTCGCCGGGTCTTTTTTg & 63 & 37 \\
\hline shRNA-NC-R & AATTCAAAAAAGACCCGGCGACGGGCTAGTC TTCAAGAGAGACTAGCCCGTCGCCGGGTCg & & \\
\hline miR-375-qF & TTTGTTCGTTCGGCTCGCGTTA & & 60 \\
\hline
\end{tabular}


Proteins were separated by SDS-polyacrylamide gel electrophoresis (PAGE), transferred to nitrocellulose membranes, probed with antibodies, and finally visualized by chemiluminescence (Millipore, Burlington, MA, USA). The antibodies used were anti-HA, anti-Flag, anti-H3, anti-GAPDH monoclonal antibody, and HRP-conjugated goat anti-mouse IgG purchased from Quintara Biosciences (Wuhan, China). The densitometric analysis of immunodetected bands was calculated as the relative bands area using Image J (NIH, Bethesda, MS, USA).

\section{Luciferase reporter assay}

The luciferase reporter assay was performed as previously described [37], with modifications. Briefly, HEK-293T cells were co-transfected with $500 \mathrm{ng}$ of the pluc- $\mathrm{M}_{\mathrm{F}} /$ pluc- $\mathrm{M}_{\mathrm{L}}$ plasmid and $500 \mathrm{ng}$ of $\mathrm{p}-\mathrm{miR}-375$ or p-miR-NC using TurboFect Transfection Reagent (Thermo Scientific) as per the manufacturer's instructions. The cells were lysed at $24 \mathrm{~h}$ after transfection, and the luciferase activity in total cell lysates was measured using the Dual-luciferase Reporter Assay System (Beyotime, Shanghai, China) according to the manufacturer's instructions.

\section{Cell cycle analysis and cell scratch assay}

Flow cytometry with propidium iodide (PI) staining was used to analyze the cell cycle progression as described previously [38]. Cells were fixed in 75\% ethanol for $2 \mathrm{~h}$, and then rehydrated in PBS at $10^{6}$ cells/mL. After they were treated with $10 \mu \mathrm{g} / \mathrm{mL}$ PI (Sigma, St. Louis, MO, USA) for $30 \mathrm{~min}$, the cells were analyzed using a flow cytometer or kept in dark conditions on ice or at $4^{\circ} \mathrm{C}$ for later use. Cell cycles were analyzed using a BD FACSCalibur Flow Cytometer with FlowJo (BD Biosciences, San Jose, CA, USA).

After transfection or pretreatment with DF-1 cells for $24 \mathrm{~h}$ in 24 -well plates, cell scratches were created using small tips at the same position in each well, and cells were cultivated in an incubator at $37^{\circ} \mathrm{C}$. After $24 \mathrm{~h}$, wound healing was observed under a microscope. The wound closure rate (expressed as a percentage) was calculated as the relative wound area using Image J (NIH, Bethesda, MS, USA).

\section{Statistical analysis}

All results are expressed as means \pm standard error of the mean (SEM). To identify significant differences, means were compared by Student's $t$-tests for viral load and mRNA levels using Padgraph. $P<$ 0.05 was considered significant.

\section{Results}

\section{NDV infection induces the up-regulation of miR-375 expression.}

Chicken embryos were infected with the velogenic F48E9 strain or lentogenic La Sota strain of NDV with a HA titer of $2^{4}$. The typical clinical lesions of F48E9 infection, like hemorrhage, were observed, but no observable lesions were observed in La Sota infected chicken embryos or the control (Figure S1A). ND virus were detected in samples infected with F48E9 or La Sota, but not in the control (Figure 1A and figure S1B,C).

A total of 98 differentially expressed miRNAs were screened from small RNA sequencing result (Figure 1B) [39]. Intergroup comparisons were used to identify changes in miRNA expression caused by NDV infection. La Sota affected the expression of 61 miRNAs (36 upregulated and 25 downregulated) at 36 hpi, and F48E9 infection altered the expression levels of 64 miRNAs (33 upregulated and 31 downregulated) (Figure 1B and Table S1). The target genes of those miRNAs were further predicted and analyzed by bioinformatics. The obtained results revealed that these miRNAs might involve in regulation cell growth, metabolism, cell cycle, immune and inflammatory responses [39].

miR-375, miR-9, miR-122, and miR-451 exhibited high expression after viral infection and had one or more predicted targets in the NDV genome or anti-genome according to an analysis using BLAST (Figures 1B, S2A). The roles of these four miRNAs in NDV infection were further evaluated in DF-1 cells by transfecting plasmids harboring the transcriptional unit of each. After $24 \mathrm{~h}$ post-transfection, DF-1 cells were infected with the F48E9 NDV strain for another $24 \mathrm{~h}$. The results showed that miR-375 resulted in the most significant reduction in NDV proliferation, followed by miR-9 (Figure 1C). The overexpression of miR-122 and miR-451 did not impact NDV growth (Figure 1C). Therefore, we focused on miR-375 in subsequent analyses.

The high level expression of miR-375 detected by small RNA sequencing was further validated by RT-qPCR. Enhanced miR-375 expressions were detected by both methods with slight difference (Figure 1D), which similar with previous reports [40, 41]. According to RT-qPCR results, miR-375 expression in all the collected tissues after NDV infection were also increased (Figure S2B). In addition, a gradual increase of miR-375 expression during NDV infection in cells was observed, F48E9, as well as La Sota infection, resulted in a similar miR-375 expression dynamics within 30 hpi (Figure 1E). 


\section{Overexpression of miR-375 inhibited NDV proliferation}

The growth characteristics of NDV were analyzed in cells with artificially modification miR-375 expression. DF-1 cells were transfected with plasmid expression miR-375 or miR-375 inhibitor, sponge-375 (Figure S1C). NDV infection was performed at $24 \mathrm{~h}$ post-transfection. The supernatants were collected at 24,36 , and 48 hpi and viral loads in the supernatants were examined by determining the TCID 50 .

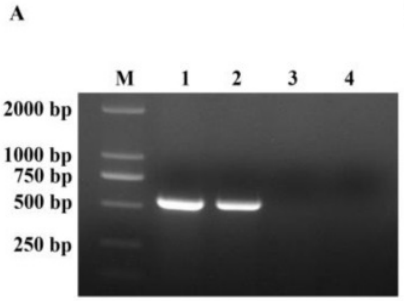

C

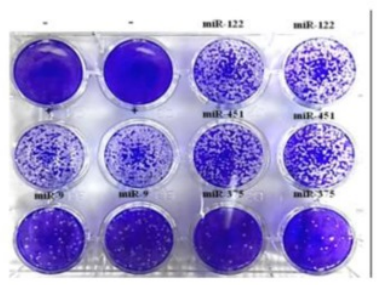

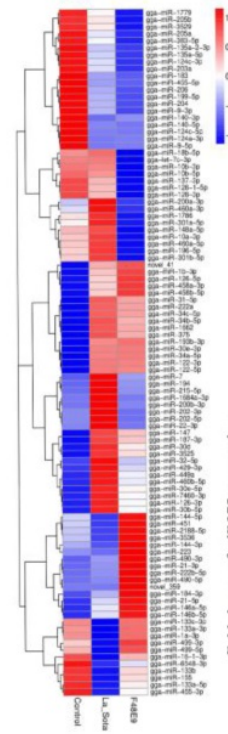

D
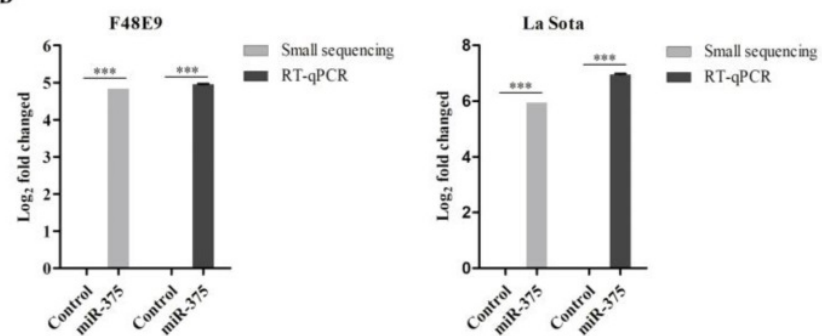

$\mathbf{E}$

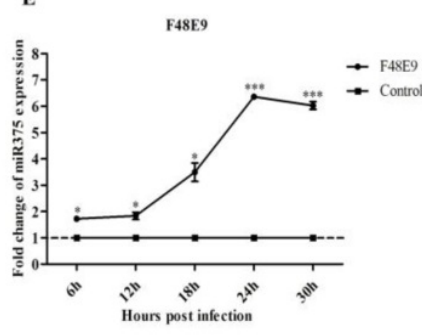

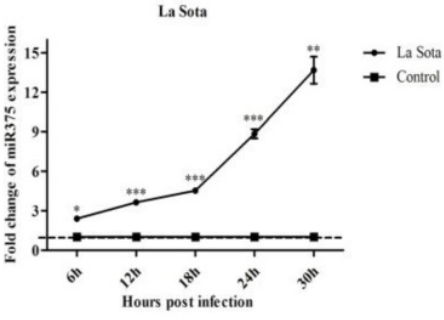

Figure 1. NDV infection up-regulated miR-375 expression in both chicken embryos and DF-1 cells. (A) PCR detected the NDV in each samples, 1-4 were representative the sample of F48E9, La Sota or PBS infection and negative control, M representative DL5000 DNA Marker. (B) The heatmap of differentially expressed miRNAs in NDV-infected chicken embryonic tissues by small RNA-Seq. (C) Plaques of F48E9 after the overexpression of miR-375, miR-9, miR-122, and miR-451. (D) miR-375 expression levels were assessed by RNA-Seq and RT-qPCR with F48E9 or La Sota infection. (E) Relative expression levels of miR-375 for various infection times were detected in DF-1 cells during F48E9 or La Sota infection. Control representative uninfected group. Statistical analyses were performed in GraphPad Prism using unpaired 2-tailed $t$-tests: $* P<0.05, * * P<0.01$, ***P $<0.001$, ns. indicates no significant difference.

A

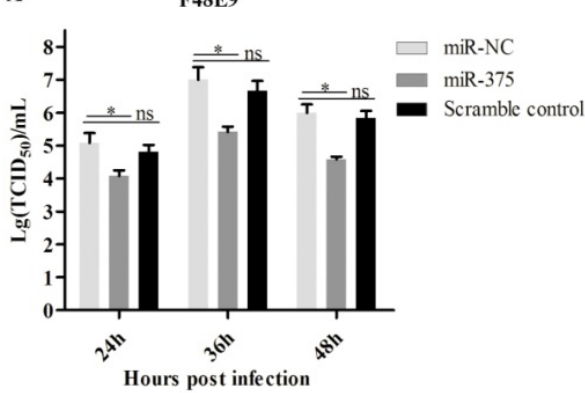

C

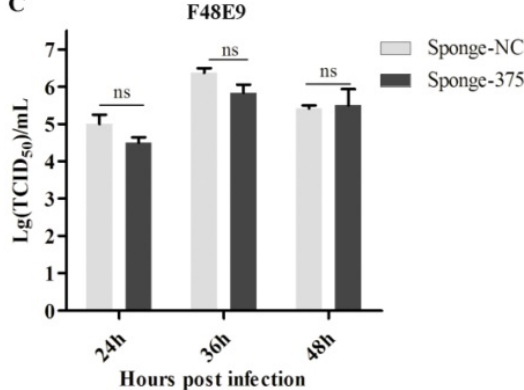

B

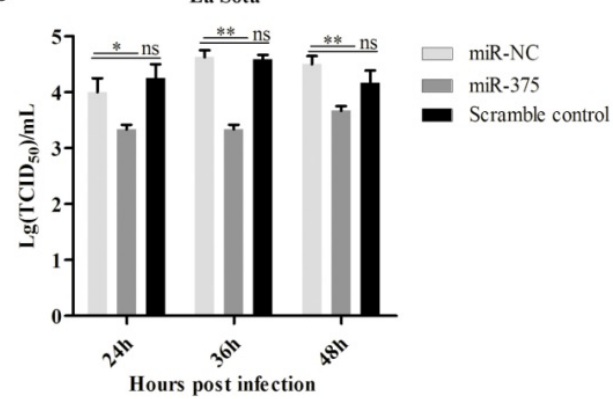

D

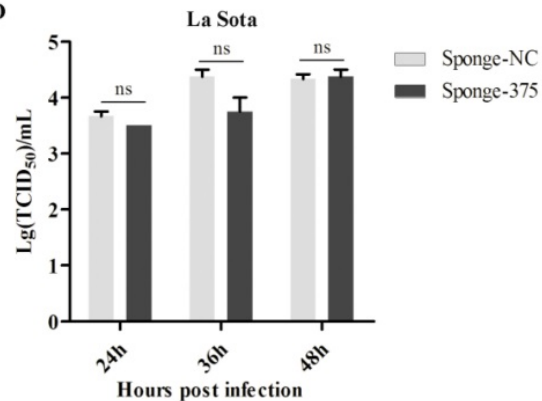

Figure 2. Antiviral of miR-375 in DF-1 cells with F48E9 or rLa Sota-GFP infections. DF-1 cells were transfected with plasmids of miR-375, sponge-375, and negative control for $24 \mathrm{~h}$, and infected with F48E9 (0.1 MOI) or rLa Sota-GFP (0.1 MOI) for another $24 \mathrm{~h}$. Viral titers of F48E9 (A, C) and rLa Sota-GFP (B, D) at various infection points were detected using the $T C I D_{50}$ method. Scramble control was mean the control with mutation in binding site of miR-375. Results are presented as means $\pm \operatorname{SEM}(n=3)$. A statistical analysis was performed in GraphPad Prism using unpaired 2-tailed $t$-tests: $* P<0.05, * * P<0.01$, ns. indicates no significant difference. 
A

3'-AUUGCGCUCGGCUUGC UU GUUU-5' miR-375

| | | | | |

La Sota (434-440) 5'-T T GT G GCAA AC AA AT A CT C AT C A GT -3

3'-A UUG C GCU C G GC UUG C U U G U U U -5' miR-375

| | | | | |

F48E9 (434-440) 5'-T C G T G G C G A A C A A A T A T T C G T C A G T -3'

C

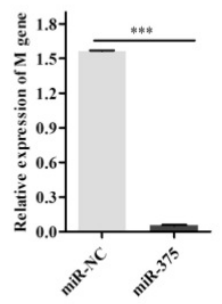

D

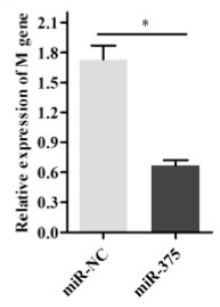

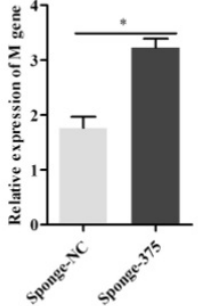

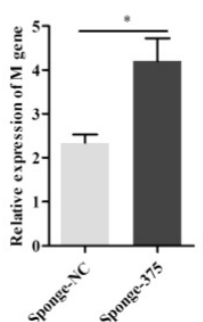

$\mathbf{E}$
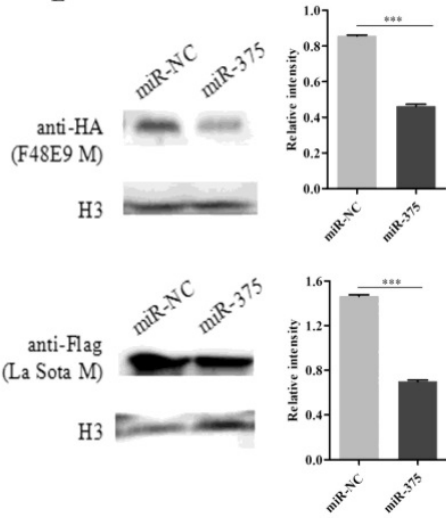

B

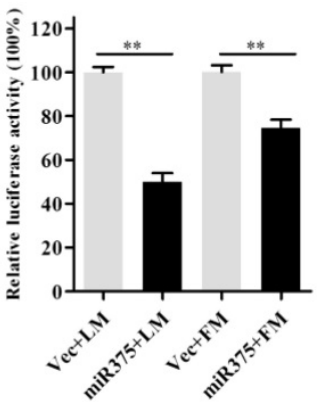

Figure 3. Degradation of $M$ gene by miR-375. (A) The target sequence of the $M$ gene was predicted using the miRanda algorithm; (B) 500 ng of F48E9 $M$ (FM) or La Sota M (LM) reporter plasmids were co-transfected with $500 \mathrm{ng}$ of miR-375 or miR-NC plasmids (Vec) into the HEK293T cells. Reporter activity was determined at $24 \mathrm{~h}$ post-transfection by Beyotime dual-luciferase assays. The plasmids of miR-375, miR-NC, sponge-375, or sponge-NC were transfected into DF- 1 cells for $24 \mathrm{~h}$, followed by F48E9 (C) or La Sota (D) infection for another $24 \mathrm{~h}$. The cells were collected to detect the M genes of rLa Sota-GFP or F48E9 by RT-qPCR. (E) Western blot analysis of the expression of M gene of F48E9 or La Sota under co-expression with miR-375 or sponge-375. Results are presented as means \pm SEM $(n=3)$. A statistical analysis was performed in GraphPad Prism using unpaired 2-tailed $t$-tests: $* p<0.05, * * p<0.01$, $* * * p<0.001$, ns. indicates no significant difference.

As shown in figure 2, the overexpression of miR-375 decreased NDV F48E9 titers by approximately 10-, 15.8- and 14.2 fold of TCID $_{50}$ at 24 , 36, and 48 hpi, respectively, as compared with the viral titers in the culture medium from infected cells transfected with miR-NC (Figure 2A). The viral titers of NDV La Sota were also reduced by 6.7-, 12.95- and 8.3 fold $\mathrm{TCID}_{50}$ at 24, 36, and 48 hpi (Figure 2B), respectively. Beyond our expectations, down-regulation miR-375 expression with miR-375 sponge, as well as antagomiR-375, did not enhance NDV proliferation (Figures 2C, D, Figure S3).

\section{miR-375 targets the $M$ gene of NDV and leads to its degradation}

The seed sequences of miR-375 were used as queries for BLAST searches against the genome and anti-genome of NDVs. One possible target site in the $\mathrm{M}$ gene (anti-genome) was identified. As shown in figure $3 \mathrm{~A}$, the seed sequences completely matched the $M$ gene (434-440) of F48E9 and six matched the M gene (434-440) of La Sota.

To further verify the relationship, a luciferase assay was performed by co-transfecting plasmids psiCheck2-FM/LM and plasmids miR-375 in HEK293T cells. It was found that luciferase activities decreased $35 \%$ and 55\% for FM and LM, respectively, when plasmid expressing miR-375 was co-transfected (Figure 3B). These results indicated that the predicted target of miR-375 within the M gene of NDV is valid. In a comparison of $\mathrm{M}$ gene sequences, we discovered that the seed sequence binding site was relatively highly conserved among NDV strains (Figure S2D).

Moreover, mRNA and protein expression of NDV $M$ gene in cells co-transfected with miR-375/sponge-375 pcDNA3.0-FM/pCAGGS-Flag-LM were also evaluated. The mRNA expression level of $M$ gene reduced by approximately 33.82-fold for F48E9 and 2.6-fold for La Sota when miR-375 was overexpressed compared with that in control cells, on the contrary, M gene (mRNA level) expression increased approximately 1.82-fold for F48E9 and 1.79-fold for La Sota with interfering miR-375 expression (Figures 3C, D). The western blot analyses were further certified that miR-375 could inhibit the expression of protein $\mathrm{M}$ (Figures 3E).

\section{Prediction of the host target of miR-375}

The increased expression of miR-375 may also impact the host. Accordingly, host targets of miR-375 were further analyzed using TargetScan. A total of 111 
targets were identified (Table S2). Among them, embryonic lethal, abnormal vision, Drosophila-like RNA binding protein 4 (ELAVL4) was a perfect target with complete seed-matching sites within the 3' UTR region (Figure 4A). As this interaction has been established previously [42], we did not perform further validation, but focused on revealing the role of ELAVL4 in NDV infection.

The complete open reading frame of ELAVL4 was cloned into a vector and the recombinant plasmids were transfected into DF-1 cells (Figure 4B). NDV infections were conducted at $24 \mathrm{~h}$ post-transfection and the supernatants were collected at 24, 36, and 48 hpi. Viral loads in the supernatants were further analyzed by evaluating the TCID 50 . Overexpression of ELAVL4 could significantly down-regulate NDV proliferation at the late infection stage, i.e., 24, 36, and 48 hpi (Figure 4C), but not at early time points, i.e., $12 \mathrm{hpi}$. As the cells transfected with the plasmid over-expressing ELAVL4 or the empty vector (Vec) looked different in growth and ELAVL4 is involved in the regulation of neuronal development according to previous analyses [43], we speculated that chicken ELAVL4 functions in the regulation of the cell cycle and influences NDV proliferation.

The cell cycle of DF-1 cells transfected with the plasmid harboring ELAVL4 was monitored by flow cytometry. The overexpression of ELAVL4 slowed cell division and proliferation, as evidenced by the decrease in cells at the S and G2 stages at $24 \mathrm{~h}$ post-transfection (Figure 4D). Cell scratch experiments were also used to analyze cell division with or without ELAVL4 overexpression. The control scratches on cells transfected with empty plasmids were repaired within $24 \mathrm{~h}$. The cells over-expressing ELAVL4 did not exhibit scratch healing at that time (Figure 4E), indicating reduced cell migration and cell cycle progression.
$\mathbf{A}$

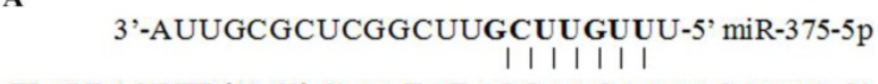

ELAVL4 3'UTR(41-60) 5'-AATATACGAACAAAACAAAA-3'
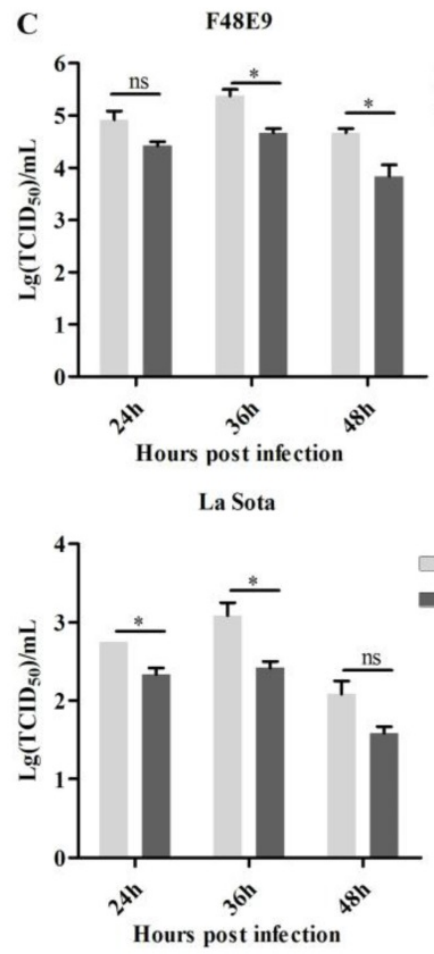

D
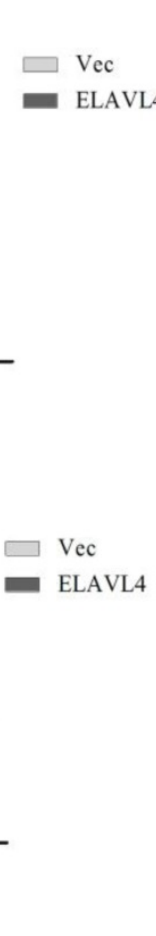

D
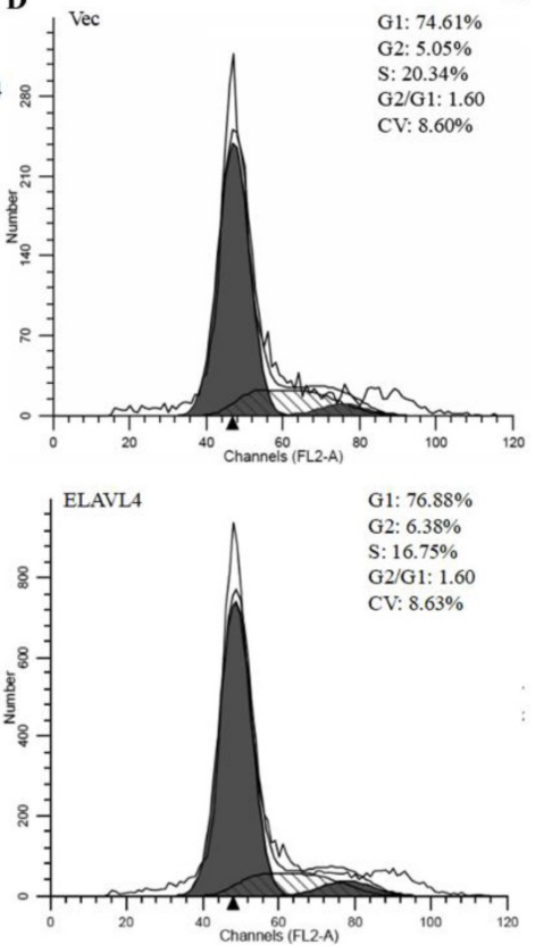

B

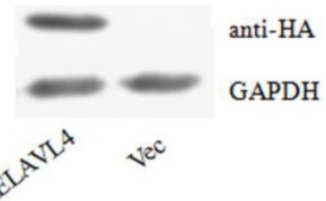

E Vec
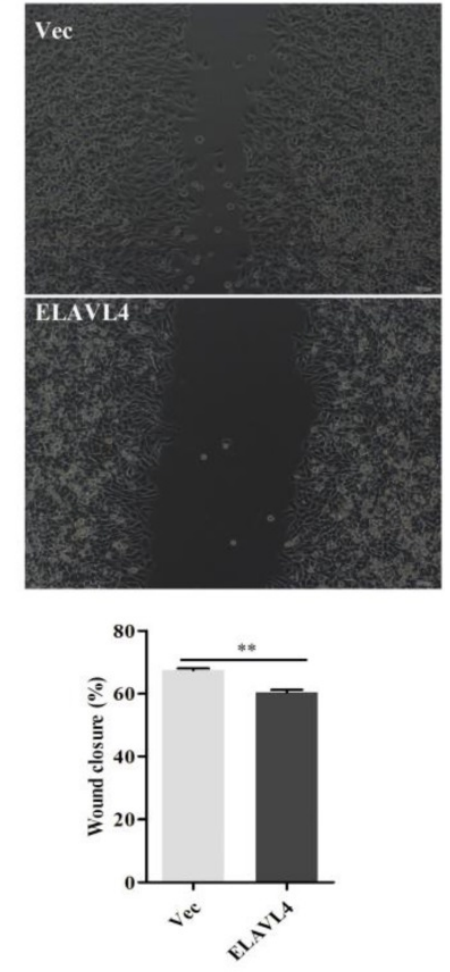

Figure 4. Antiviral function of ELAVL4 as a miR-375 host target. (A) The target sequence of the ELAVL4 gene was predicted using the miRanda algorithm. (B) The expression of ELAVL4 was detected by western blotting. (C) DF-1 cells were transfected with PCAGGS-HA-ELAVL4 and vector plasmids (Vec) for $24 \mathrm{~h}$, and infected with F48E9 $(0.1 \mathrm{MOI})$ or rLa Sota-GFP $(0.1 \mathrm{MOI})$. The viral titers of F48E9 and rLa Sota-GFP at various infection points were detected using the TCID 50 method. (D) Flow cytometry was performed to analyze changes in the cell cycle after ELAVL4 overexpression and vector (Vec) transfection. (E) A scratch assay was used to verify cell growth indirectly after ELAVL4 overexpression and vector $(\mathrm{Vec})$ transfection. The wound closure percentage was calculated as the relative wound area using Image J. Results are presented as means $\pm \operatorname{SEM}(n=3)$. Statistical analyses were performed in GraphPad Prism using unpaired 2-tailed $t$-tests: $* P<0.05$, $* * P$ $<0.01$, ns. indicates no significant difference. 

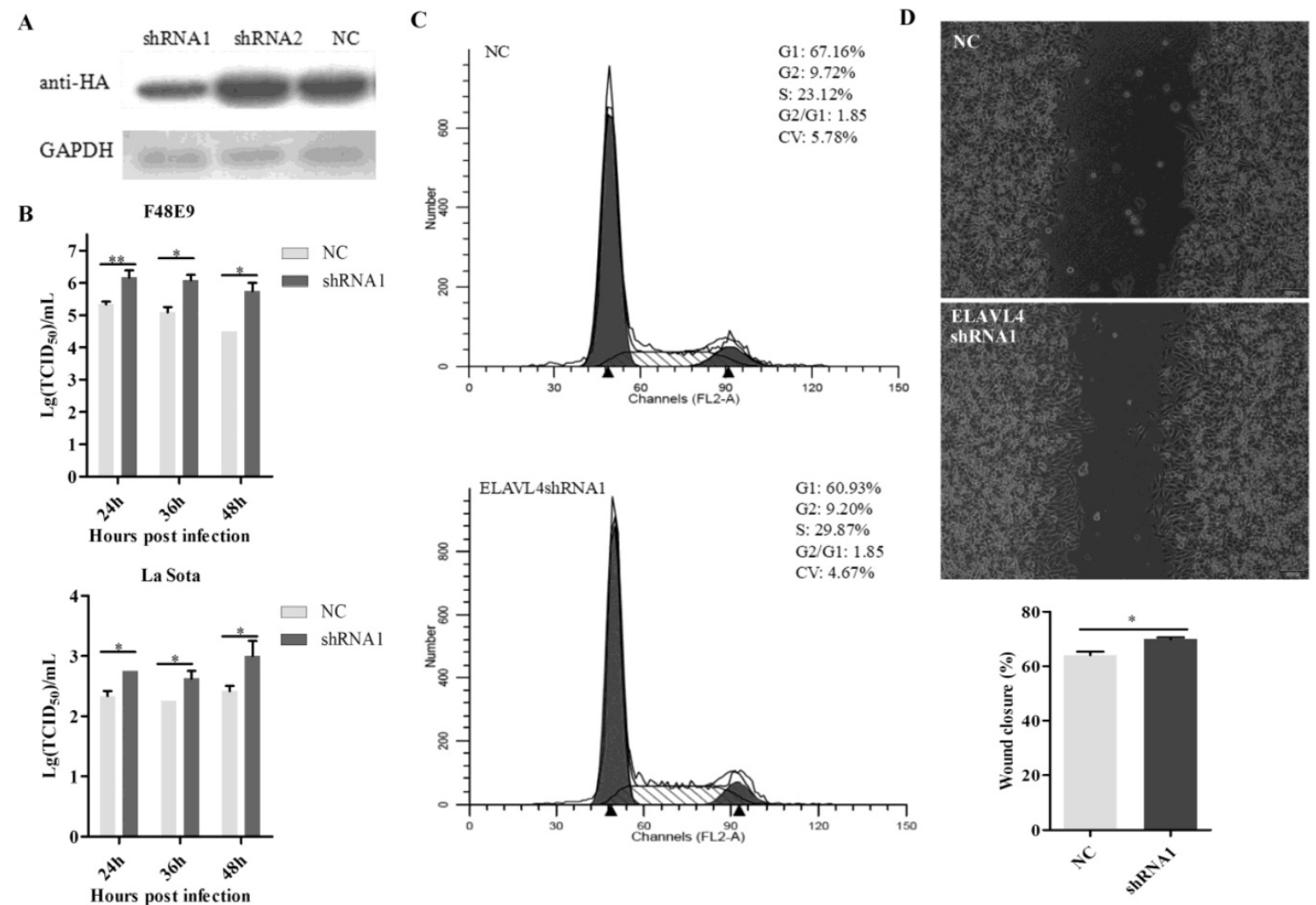

Figure 5. Interference of ELAVL4 could promote NDV proliferation and cell growth. (A) The efficiency of interference of shRNA targeting ELAVL4 was tested by western blotting. (B) DF-1 cells were transfected with ELAVL4-shRNAl and shRNA-NC plasmids for 24 h, and infected with rLa Sota-GFP or F48E9 (0.1 MOI). The viral titers of F48E9 or rLa Sota-GFP at various infection points were detected using the TCID 50 method. (C) Flow cytometry was performed to analyze changes in the cell cycle after ELAVL4-shRNAl or NC plasmid transfection for $24 \mathrm{~h}$. (D) A scratch assay was used to verify cell growth indirectly after ELAVL4-shRNA1 or NC plasmid transfection for $24 \mathrm{~h}$. The wound closure percentage was calculated as the relative wound area using Image J. Results are presented as means \pm SEM $(n=3)$. Statistical analyses were performed in GraphPad Prism using unpaired 2-tailed $t$-tests: $* P<0.05$, $* * P<0.01$.

The shRNAs targeting ELAVL4 (sh-ELAVL4) were designed to down-regulate its expression in DF-1 cells. Based on a western blot assay, shRNA1 was more effective than shRNA2 with respect to reducing ELAVL4 expression (Figure 5A). Therefore, shRNA1 was used in further studies. A plasmid expressing shRNA1, p-shRNA1, was transfected into DF-1 cells. NDV growth in cells transfected with p-shRNA1 was also detected. The shRNA-mediated down-regulation of ELAVL4 significantly enhanced both velogenic NDV F48E9 and lentogenic NDV La Sota proliferation in DF-1 cells (Figure 5B). The cell cycles for cells with or without ELAVL4 down-regulation by shRNA were monitored by flow cytometry. The down-regulation of ELAVL4 activated cell division, as evidenced by the increase in cells transfected with p-shRNA1 in the $\mathrm{S}$ and G2 stages (Figure 5C). Moreover, accelerated cell scratch healing also demonstrated rapid cell proliferation in the cell scratch assay (Figure 5D) when ELAVL4 was artificially downregulated by shRNA.

\section{miR-375 regulated bystander cells}

We demonstrated counteracting roles of miR-375 in NDV infection. However, in uninfected cells, miR-375 promotes cell proliferation, which is beneficial for viruses, as more hosts are available in this situation. If miR-375 affects bystander cells surrounding the infected cells, it may have additional implications for NDV. As reported previously, microRNAs are secreted from cells via exosomes; we speculated that miR-375 could also be secreted from infected cells and influence the growth rate of bystander cells.

To evaluate this hypothesis, DF-1 cells were transfected with p-miR-375 and RT-qPCR was used to quantify miR-375 in cells and supernatants. Significantly higher levels of miR-375 were detected in cells transfected with p-miR-375 (Figure 6A) and the corresponding supernatants than in untransfected controls (Figure 6B), indicating that miR-375 was secreted from the cells. Additionally, miR-375 in the supernatants decreased to normal levels within $48 \mathrm{~h}$ at $37^{\circ} \mathrm{C}$ (Figure 6C). 
A
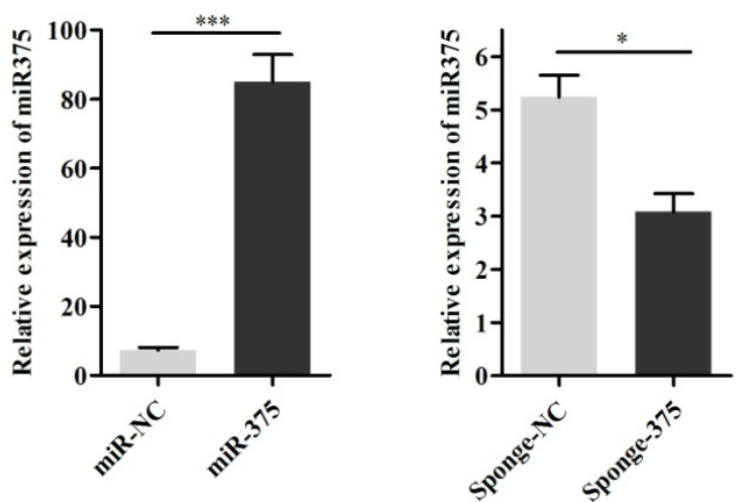

B
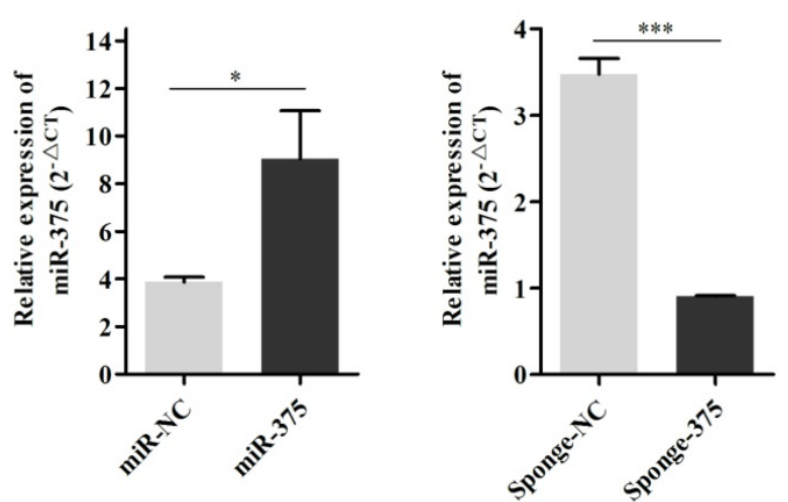

C

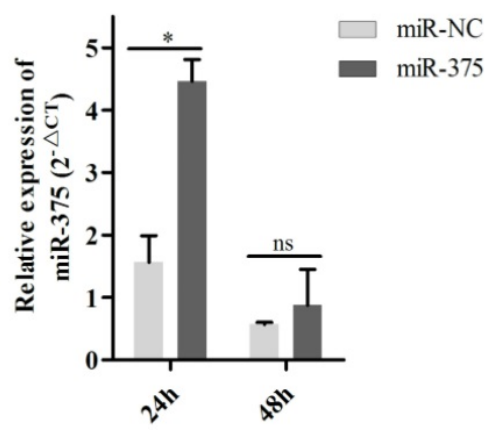

Figure 6. Secretion of miR375 into the supernatant. miR-375 levels in cells $(\mathbf{A})$ and supernatants (B) after Hep2 cell transfection with the plasmid miR-375, miR-NC sponge-375, or sponge-NC for $24 \mathrm{~h}$. (C) miR-375 was degraded in the supernatant at $37^{\circ} \mathrm{C}$ for $24 \mathrm{~h}$ or $48 \mathrm{~h}$ by RT-qPCR. Results are presented as means \pm SEM $(n=3)$. Statistical analyses were performed in GraphPad Prism using unpaired 2-tailed t-tests: $* p<0.05$, $* * p<0.01$, $* * * p<$ $0.001, \mathrm{~ns}$. indicates no significant difference.

\section{NDV replication differs between cells treated with miR-375 pre- or post-viral inoculation}

The supernatants containing miR-375 were collected at $24 \mathrm{~h}$ from Hep2 cells transfected with p-miR-375. DF-1 cells in 24-well plates were inoculated with NDV; after $1 \mathrm{~h}$, the cells were washed with PBS and $500 \mu \mathrm{L}$ of the supernatant was added to the wells to treat the infected cells. Viral loads in the medium at $24 \mathrm{~h}$ post-inoculation were quantified by counting fluorescent cells (La Sota, Figures 7A, B) or plaques (F48E9, Figures 7C, D). As predicted, cells with supernatants containing miR-375 significantly reduced F48E9 and La Sota proliferation (Figure 7).

Then, DF-1 cells were pre-treated with the supernatants from Hep2 cells for $24 \mathrm{~h}$ and infected with La Sota or F48E9 (0.1 MOI). Similarly, viral loads in the culture media at $24 \mathrm{hpi}$ were quantified by counting fluorescent cells (Figures 8A, B) or plaques (Figures 8C, D). The results showed that the proliferation rates of both La Sota and F48E9 were enhanced by pre-treating cells with supernatants containing miR-375 (Figure 8). Both flow cytometry assays (Figure 9A) and cell scratch assays (Figure 9B) indicated its enhanced cell growth.

\section{Discussion}

MiR-375 was down-regulated in various oncogenic diseases or cancer types according to microarray and deep sequencing analyses [44-46]. Yet, it was up-regulated during NDV infection, an oncolytic virus infection. This finding indicated that miR-375 may be useful for NDV-mediated cancer therapy $[46,47]$. In the current sturdy, we found that La Sota, but not F48E9, infection induces increased levels of miR-375 expression. These results suggested that the up-regulation of miR-375 expression is a slow process. F48E9 failed to induce a higher level of miR-375 expression, possibly because velogenic NDV killed cells too rapidly. Fittingly, La Sota is used more often than F48E9 in cancer therapy.

MiR-375 had an antiviral effect during NDV infection by targeting the $\mathrm{M}$ gene of $\mathrm{NDV}$, resulting in gene degradation. The $M$ protein of NDVs is a multi-functional protein involved in virion assembly and budding from the cell membrane [30, 48]. Therefore, reduced $\mathrm{M}$ protein expression is expected to reduce virus production and release. A similar phenomenon has been reported previously; miR-485 decreases AIV replication and it has a targeting effect via viral BP1 proteins [17]. However, in the current study, the down-regulation of miR-375 expression did not promote NDV growth. This may explained by the very low level of endogenic miR-375 expression in normal cells. MiR-375 copies increased from about 27.86 in non-infected tissues to 629.06 and 1419.59 after F48E9 and La Sota infection based on small RNA-seq (Table S1). Eliminating them would have no significant effect on NDV replication. Furthermore, cells have very strong compensatory mechanisms. Each gene may controlled by hundreds of miRNAs, and each miRNA may target hundreds of genes [49]. Therefore, it is easy to understand the unexpected results in the context of the large number of regulatory factors that can offset or mask the effects on NDV proliferation. 
A
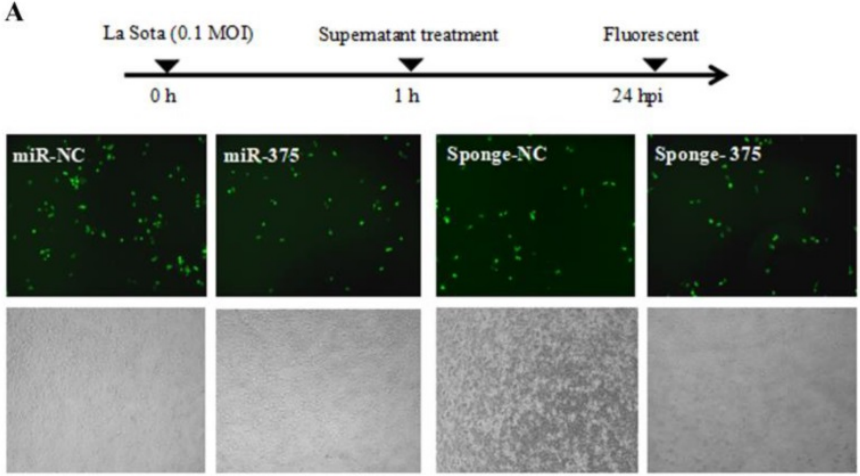

C

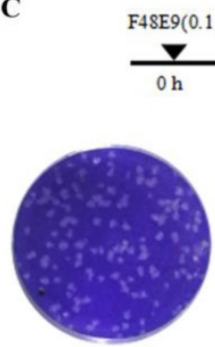

miR-NC

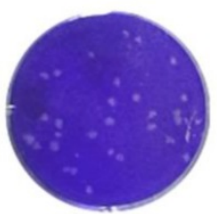

miR-375
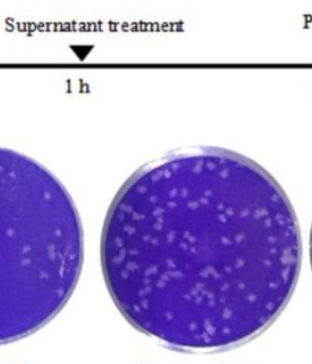

Sponge-NC
Plaque
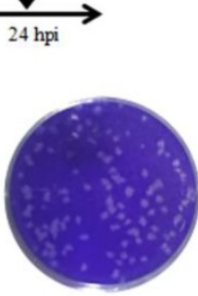

Sponge-375
B

The average count of green fluorescent cells in each microscopic filed
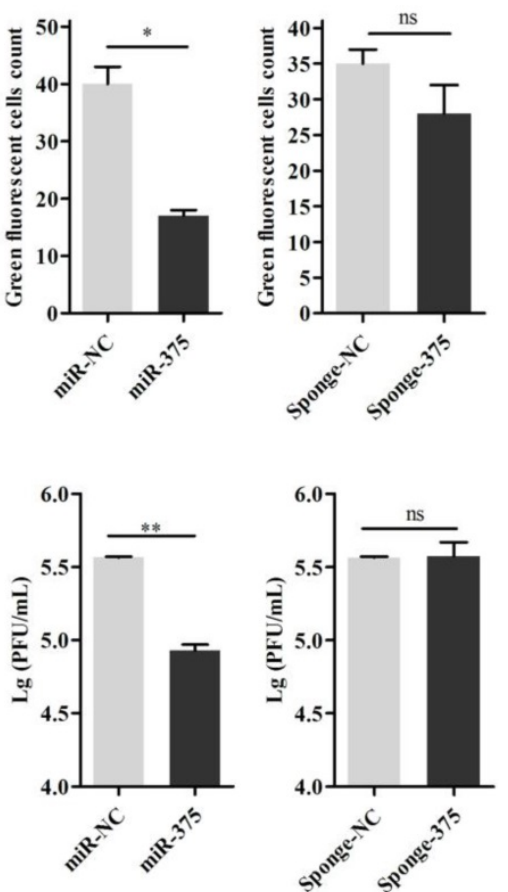

Figure 7. Treatment of DF-1 cells with miR-375 inhibit NDV replication. The supernatants containing miR375, miR-NC, sponge-375, or sponge-NC were used to culture the DF-1 cells after rLa Sota-GFP or F48E9 was incubated for $1 \mathrm{~h}$. Then, the supernatants were collected for the detection of viral titers at $24 \mathrm{~h}$. A fluorescence microscope was used for observations (A). The average count of fluorescent cells in each microscopic field was calculated (B) for rLa Sota-GFP. Plaques of F48E9 were observed (C) and counted (D). Results are presented as means \pm SEM $(n=3)$. Statistical analyses were performed in GraphPad Prism using unpaired 2-tailed t-tests: $* P<0.05, * * P<0.01$, ns. indicates no significant difference.

A
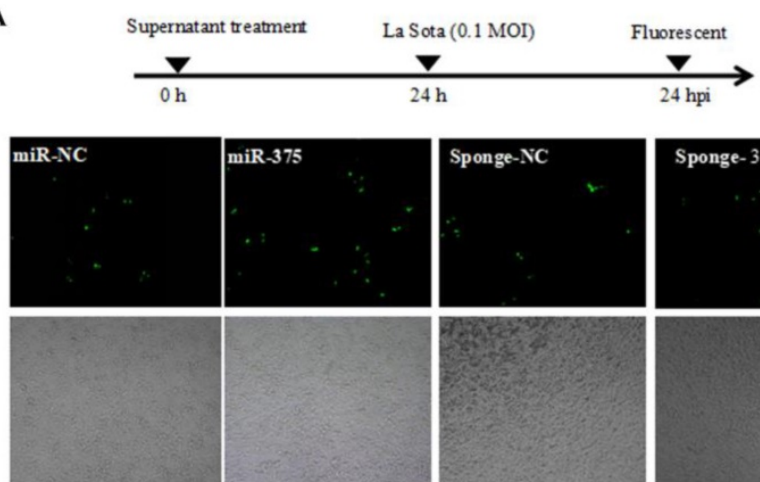

C

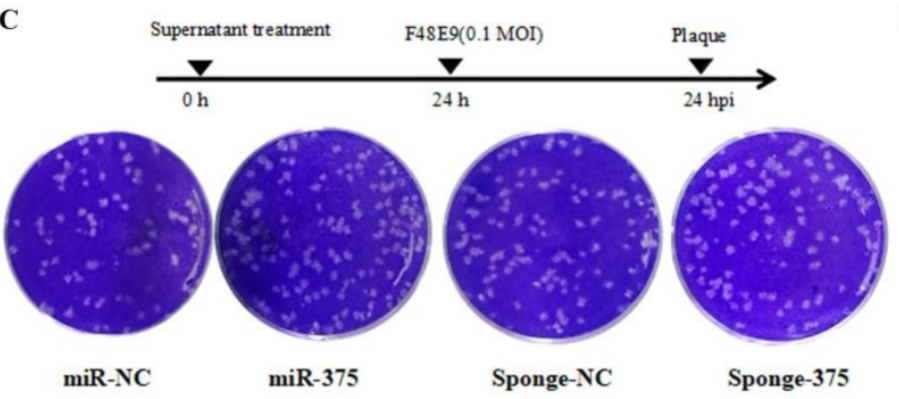

The average count of green fluorescent cells in each microscopic filed
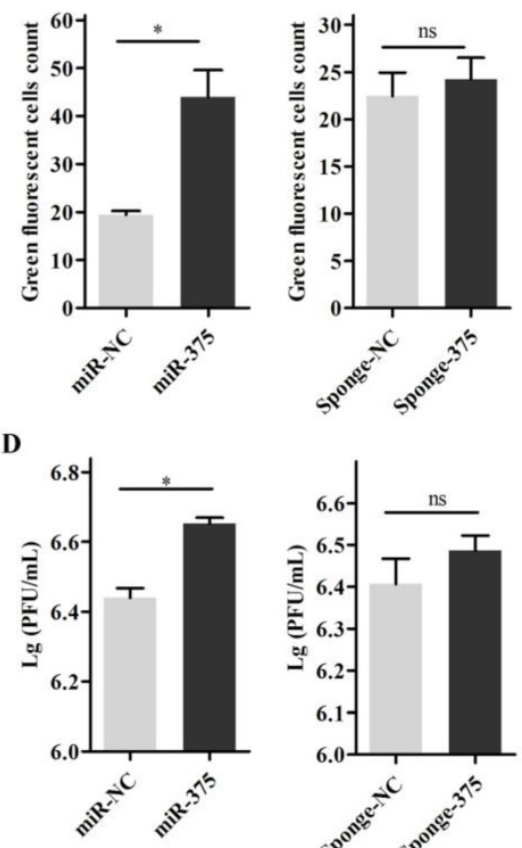

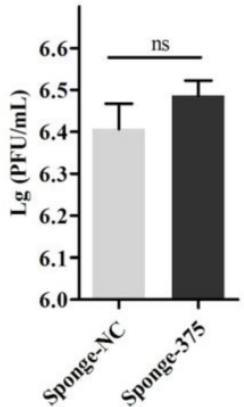

Figure 8. Pre-treatment of DF-1 cells with miR-375 has different effects on NDV proliferation. Supernatants containing miR-375, miR-NC, sponge-375, or sponge-NC were used to culture the DF-1 cells at $24 \mathrm{~h}$ before incubation with rLa Sota-GFP or F48E9. After another $24 \mathrm{~h}$ post-NDV infection, the viral titers were evaluated. Observations (A) and quantitative analyses (B) of fluorescent cells infected with rLa Sota-GFP, and observations (C) and statistical analyses (D) for the plaque assay for F48E9 infection are shown. Results are presented as means \pm SEM $(n=3)$. Statistical analyses were performed in GraphPad Prism using unpaired 2-tailed $t$-tests: $* P<0.05$, ns. indicates no significant difference. 
A
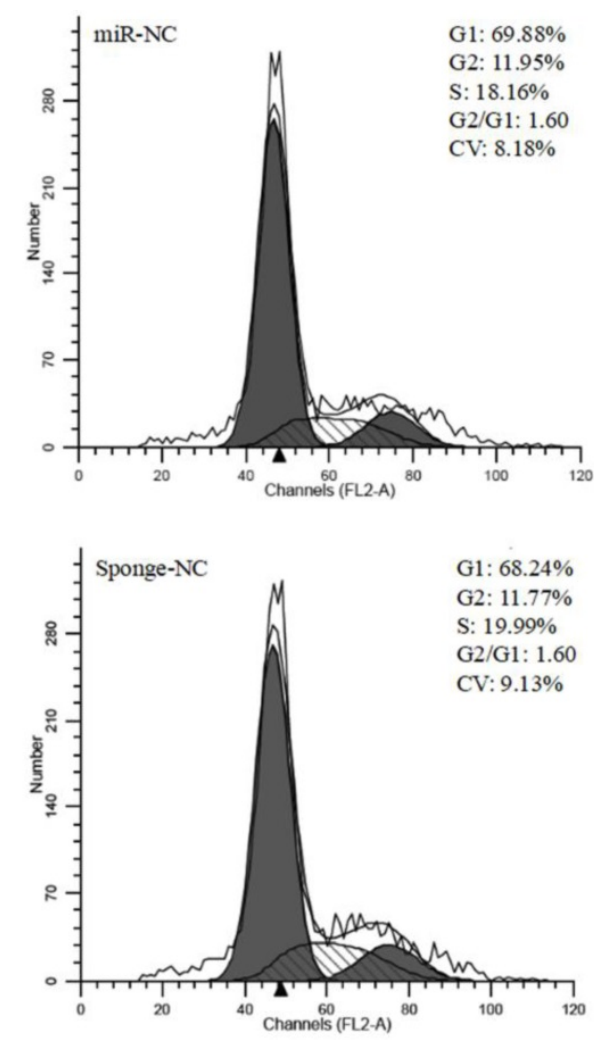
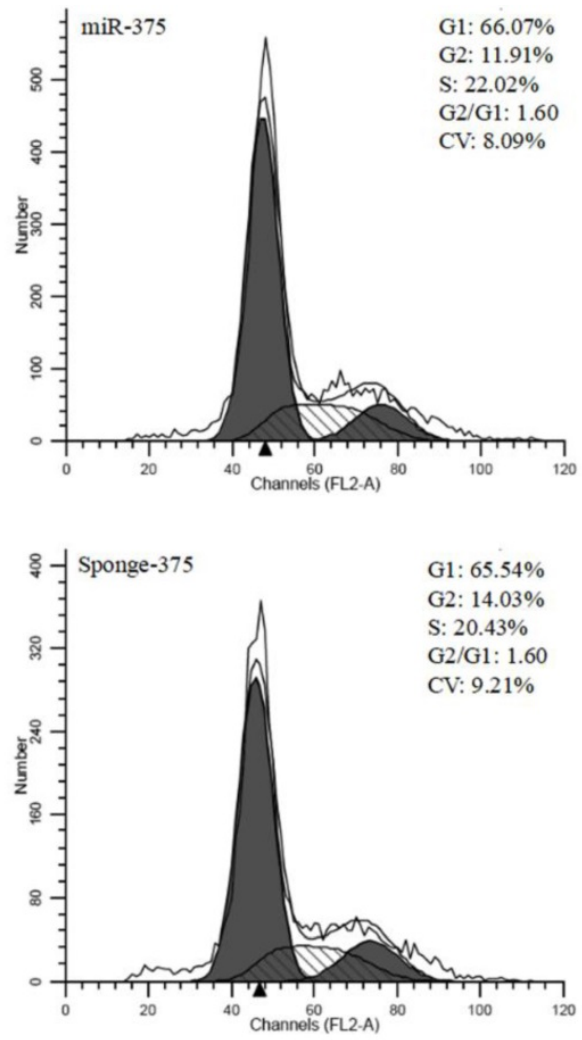

B
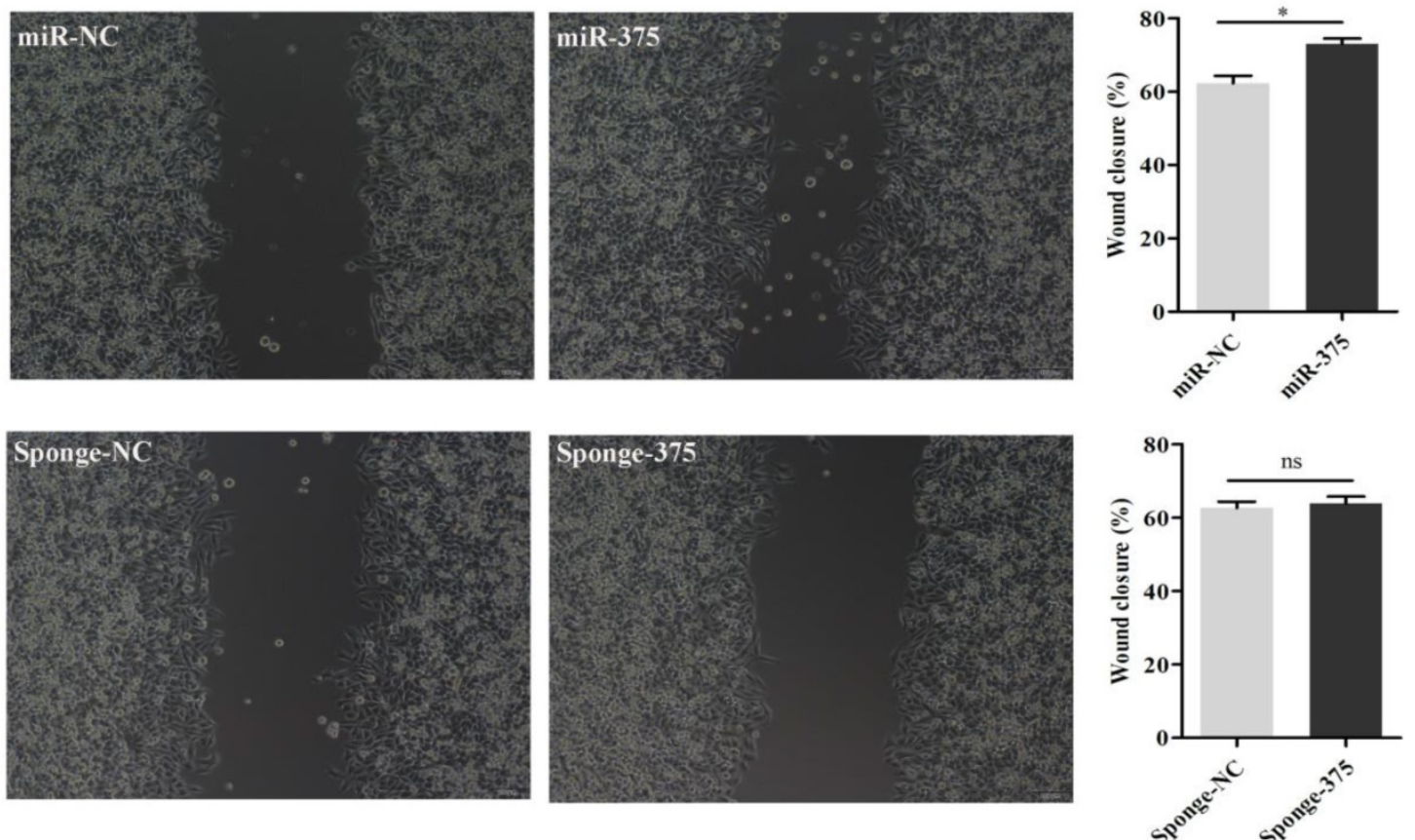

Figure 9. Analysis of the cell cycle and wound healing for DFI cells pretreated with miR-375 or sponge-375. Cell cycle analyses (A) and scratch assays (B) were performed using miR-375, miR-NC, sponge-375, or sponge-NC pretreated DF1 cells. The wound closure percentage was calculated as the relative wound area using Image J. Results are presented as means \pm SEM $(n=3)$. Statistical analyses were performed in GraphPad Prism using unpaired 2 -tailed $t$-tests: $* P<0.05$, ns. indicated no significant difference.

One of the most impressive findings in our study is the demonstration of secretory microRNA-related regulation of the cell cycle. miRNAs secreted via extracellular vesicles can act a signaling factors and mediate cell communication [50,
51]. NDV infection upregulates miR-375 expression in infected cells, and miR-375 secreted by infected cells can mediate the growth of surrounding cells. The enhanced cell growth mediated by secretory miR-375 is beneficial for viral growth. However, miR-375 also 
has a target in the viral genome. The similar proliferation characteristics of DF-1 cells transfected with plasmids over-expressing miR-375 or treated with the supernatants from Hep2 cells transfected with the plasmid p-miR-375 indicate that miR-375, but not other components, was the functional element. In addition, similar results were obtained for NDV proliferation on cells treated with the transfection plasmid or supernatants from Hep2 cells. As miR-375 in the supernatant from transfected cells is ephemeral, it influences cells, but not NDV. Therefore, cells pretreated with supernatants exhibited enhanced virus proliferation. In addition, a degradation test indicated that miR-375 was significantly reduced at 48 $\mathrm{h}$ in the supernatant under $5 \% \quad \mathrm{CO}_{2}$ and $37^{\circ} \mathrm{C}$. However, additional studies are needed to determine how miR-375 is released from transfected Hep2 cells.

The effect of external secretion from infected cells has also been identified previously, such as EV or paracrine effects [50, 52, 53]. It is considered that miRNAs secreted in EVs can be transferred to target cells, and directly modulate target genes [51]. For example, viral miRNAs could be secreted from Epstein-Barr virus-infected cells into non-infected cells, resulting in the suppression of viral target genes [52]. Infection activates IFN production and the secretory IFNs have antiviral effects via combining with the IFN-alpha/beta receptors on the cell surface, thereby activating the JAK-STAT signaling cascade [54]. Then, hundreds of interferon-stimulated genes are expressed to establish an antiviral state after pathway activation in the cells [55, 56]. Via these pathways, the uninfected cells could establish an antiviral state, without exposure to the virus. The paracrine effect of inflammatory cytokines also plays an important role in the activation of the inflammatory response $[57,58]$. Here, we found that miR-375 could be secreted from uninfected cells, affecting NDV infection. These findings suggest that miRNAs may play a larger role in vivo than has been established owing to the effects of their secretion. In previous studies, the functions of miRNAs in infection are frequently evaluated based on a single-step infection analysis. The results of our study indicated that the roles of miRNAs may be distinct during progressive rounds of virus transmission.

ELAVL4, an RNA binding protein, is associated with neurodevelopment and diseases, such as neuronal cell maturation, malignant neuroblastoma, and Parkinson's disease [43, 59-61]. ELAVL4 is related to pre-mRNA processing or the enhancement of translation via interactions with eIF4A $[62,63]$. An overexpression experiment demonstrated that $\mathrm{HuD}$ functions in the initiation of neurite outgrowth via, at least in part, its regulation of GAP-43 expression [64].
ELAVL4 depletion decreased the proliferation of A549 NSCLC cells, a non-small cell lung cancer cell line, and enhanced the radiation sensitivity of the cells, possibly by increasing apoptotic cell death [65]. Contrary to expectations, flow cytometry and scratch-healing assays revealed that the overexpression of ELAVL4 decreased DF-1 cell proliferation. Knocking-down ELAVL4 by shRNA accelerated DF-1 cell growth. Our data provide novel insights into the function of ELAVL4 in normal chicken cells. In future studies, it will be interesting to examine whether ELAVL4 plays different roles in different species and/or tissues. The evolutionary diversification of the HuD/ELAV-like RBP gene family in insects has been identified previously [66].

In the current study, we found that miR-375 could significant reduce NDV proliferation by targeting the M gene of NDV. We also discovered that miR-375 could be externally secreted from DF-1 cells. The released miR-375 could regulate the growth rate of treated cells by targeting ELAVL4 and could influence the proliferation of the virus in the cells. Our results provide novel insights into the function of miRNAs in the regulation of viral infection.

\section{Abbreviations}

NDV: Newcastle disease virus; ELAVL4: embryonic lethal, abnormal vision, Drosophila-like RNA binding protein 4; AGO2: Argonaute proteins; DF-1: chicken embryo fibroblast cells; HA: hemagglutination assay; PFU: plaque forming unit; MOI: multiplicity of infection; $\mathrm{TCID}_{50}: 50 \%$ tissue culture infectious dose; PI: propidium iodide; EV: extracellular vesicles.

\section{Supplementary Material}

Supplementary figures and tables. http://www.ijbs.com/v15p0044s1.pdf

\section{Acknowledgements}

We thank most sincerely Yanhong Wang and Wenbin Wang (Northwest A\&F University) for material presentation. This work was supported by grants from the National Natural Science Foundation of China (31672581 for XLW and 31572538 for ZQY).

\section{Competing Interests}

The authors have declared that no competing interest exists.

\section{References}

1. Bartel DP. MicroRNAs: genomics, biogenesis, mechanism, and function. Cell. 2004; 116: 281-97.

2. Esquela-Kerscher A, Slack FJ. Oncomirs - microRNAs with a role in cancer. Nature reviews Cancer. 2006; 6: 259-69. 
3. Ouyang W, Wang YS, Du XN, Liu HJ, Zhang HB. gga-miR-9* inhibits IFN production in antiviral innate immunity by targeting interferon regulatory factor 2 to promote IBDV replication. Veterinary microbiology. 2015; 178: 41-9.

4. Lee YAC, Han J, Choi H, Kim J, Yim J, Lee J, Provost P, Rådmark O, Kim S, Kim VN. The nuclear RNase III Drosha initiates microRNA processing. Mature. 2003; 425: 415-9.

5. Kim VN. MicroRNA precursors in motion: exportin-5 mediates their nuclear export. Trends Cell Biol. 2004; 14: 156-9.

6. Yi R, Qin Y, Macara IG, Cullen BR. Exportin-5 mediates the nuclear export of pre-microRNAs and short hairpin RNAs. Gene Dev. 2003; 17: 3011-6.

7. Bohnsack MT, Czaplinski K, Gorlich D. Exportin 5 is a RanGTP-dependent dsRNA-binding protein that mediates nuclear export of pre-miRNAs. Rna. 2004; 10: 185-91.

8. Lund E, Guttinger S, Calado A, Dahlberg JE, Kutay U. Nuclear export of microRNA precursors. Science. 2004; 303: 95-8.

9. Ha M, Kim VN. Regulation of microRNA biogenesis. Nat Rev Mol Cell Bio. 2014; 15: 509-24.

10. Kulkarni M, Ozgur S, Stoecklin G. On track with P-bodies. Biochem Soc T. 2010; 38: 242-51.

11. Ouyang H, He X, Li G, Xu H, Jia X, Nie Q, et al. Deep Sequencing Analysis of miRNA Expression in Breast Muscle of Fast-Growing and Slow-Growing Broilers. International journal of molecular sciences. 2015; 16: 16242-62.

12. Bandiera S, Pernot S, El Saghire H, Durand SC, Thumann C, Crouchet E, et al. Hepatitis C Virus-Induced Upregulation of MicroRNA miR-146a-5p in Hepatocytes Promotes Viral Infection and Deregulates Metabolic Pathways Associated with Liver Disease Pathogenesis. J Virol. 2016; 90: 6387-400.

13. Lin J, Xia J, Chen YT, Zhang KY, Zeng Y, Yang Q. H9N2 avian influenza virus enhances the immune responses of BMDCs by down-regulating miR29c. Vaccine. 2017; 35: 729-37.

14. Yu Z, Gao X, Liu C, Lv X, Zheng S. Analysis of microRNA expression profile in specific pathogen-free chickens in response to reticuloendotheliosis virus infection. Applied microbiology and biotechnology. 2017; 101: 2767-77.

15. Conrad KD, Giering F, Erfurth C, Neumann A, Fehr C, Meister G, et al. microRNA-122 Dependent Binding of Ago2 Protein to Hepatitis C Virus RNA Is Associated with Enhanced RNA Stability and Translation Stimulation. Plos One. 2013; 8: e56272.

16. Zheng ZH, Ke XL, Wang M, He SY, Li Q, Zheng CS, et al. Human MicroRNA hsa-miR-296-5p Suppresses Enterovirus 71 Replication by Targeting the Viral Genome. Journal of Virology. 2013; 87: 5645-56.

17. Ingle H, Kumar S, Raut AA, Mishra A, Kulkarni DD, Kameyama T, et al. The microRNA miR-485 targets host and influenza virus transcripts to regulate antiviral immunity and restrict viral replication. Sci Signal. 2015; 8: ra126.

18. Thibault PA, Huys A, Amador-Canizares Y, Gailius JE, Pinel DE, Wilson JA. Regulation of Hepatitis C Virus Genome Replication by Xrn1 and MicroRNA-122 Binding to Individual Sites in the 5 ' Untranslated Region. Journal of Virology. 2015; 89: 6294-311.

19. Jopling CL. Regulation of hepatitis C virus by microRNA-122. Biochem Soc T. 2008; 36: 1220-3.

20. Conrad KD, Giering F, Erfurth C, Neumann A, Fehr C, Meister G, et al. microRNA-122 Dependent Binding of Ago2 Protein to Hepatitis C Virus RNA Is Associated with Enhanced RNA Stability and Translation Stimulation (vol 8, e56272, 2013). Plos One. 2016; 11: e56272.

21. Wen WT, He ZJ, Jing QL, Hu YW, Lin CJ, Zhou R, et al. Cellular microRNA-miR-548g-3p modulates the replication of dengue virus. J Infection. 2015; 70: 631-40.

22. Wang LL, Qin Y, Tong L, Wu S, Wang Q, Jiao QG, et al. MiR-342-5p suppresses coxsackievirus $\mathrm{B} 3$ biosynthesis by targeting the $2 \mathrm{C}$-coding region. Antivir Res. 2012; 93: 270-9.

23. Harden ME, Prasad N, Griffiths A, Munger K. Modulation of microRNA-mRNA Target Pairs by Human Papillomavirus 16 Oncoproteins. mBio. 2017; 8: e02170-16.

24. Dortmans JC, Koch G, Rottier PJ, Peeters BP. Virulence of Newcastle disease virus: what is known so far? Veterinary research. 2011; 42 : 122

25. Chen S, Hao H, Liu Q, Wang R, Zhang P, Wang X, et al. Phylogenetic and pathogenic analyses of two virulent Newcastle disease viruses isolated from Crested Ibis (Nipponia nippon) in China. Virus genes. 2013; 46: 447-53.

26. Brown VR, Bevins SN. A review of virulent Newcastle disease viruses in the United States and the role of wild birds in viral persistence and spread. Veterinary research. 2017; 48: 68.

27. Ganar K, Das M, Sinha S, Kumar S. Newcastle disease virus: current status and our understanding. Virus research. 2014; 184: 71-81.

28. Cox RM, Plemper RK. Structure and organization of paramyxovirus particles. Current opinion in virology. 2017; 24: 105-14.

29. Noton SL, Fearns R. Initiation and regulation of paramyxovirus transcription and replication. Virology. 2015; 479-480: 545-54.

30. Xu H, Duan Z, Chen Y, Liu J, Cheng X, Liu J, et al. Simultaneous mutation of G275A and P276A in the matrix protein of Newcastle disease virus decreases virus replication and budding. Archives of virology. 2016; 161: 3527-33.

31. Wang Y, Bi Y, Yu W, Wei N, Wang W, Wei Q, et al. Two mutations in the HR2 region of Newcastle disease virus fusion protein with a cleavage motif "RRQRRL" are critical for fusogenic activity. Virology journal. 2017; 14: 185

32. Lewis BP, Shih IH, Jones-Rhoades MW, Bartel DP, Burge CB. Prediction of mammalian microRNA targets. Cell. 2003; 115: 787-98.

33. Pizzi M. Sampling variation of the fifty percent end-point, determined by the Reed-Muench (Behrens) method. Human biology. 1950; 22: 151-90.
34. Qiu XS, Zhan Y, Meng CC, Wang JQ, Dong LN, Sun YJ, et al. Identification and functional analysis of phosphorylation in Newcastle disease virus phosphoprotein. Archives of virology. 2016; 161: 2103-16.

35. Chia SL, Yusoff $K$, Shafee N. Viral persistence in colorectal cancer cells infected by Newcastle disease virus. Virology journal. 2014; 11: 91.

36. Feng XX, Wang Z, Shi JZ, Deng GH, Kong HH, Tao SY, et al. Glycine at Position 622 in PB1 Contributes to the Virulence of H5N1 Avian Influenza Virus in Mice. Journal of Virology. 2016; 90: 1872-9.

37. Xiao SQ, Wang X, Ni HB, Li N, Zhang AK, Liu HL, et al. MicroRNA miR-24-3p Promotes Porcine Reproductive and Respiratory Syndrome Virus Replication through Suppression of Heme Oxygenase-1 Expression. Journal of Virology. 2015; 89: 4494-503.

38. Huang QC, Yun XM, Rao WB, Xiao CY. Antioxidative cellular response of lepidopteran ovarian cells to photoactivated alpha-terthienyl. Pestic Biochem Phys. 2017; 137: 1-7.

39. Jia YQ, Wang $\mathrm{XL}$, Wang $\mathrm{XW}$, Yan $\mathrm{CQ}, \mathrm{Lv} \mathrm{CJ}, \mathrm{Li} \mathrm{XQ}$, et al. Common microRNA(-)mRNA Interactions in Different Newcastle Disease Virus-Infected Chicken Embryonic Visceral Tissues. International journal of molecular sciences. 2018; 19: E1291.

40. Ranaware PB, Mishra A, Vijayakumar P, Gandhale PN, Kumar H, Kulkarni DD, et al. Genome Wide Host Gene Expression Analysis in Chicken Lungs Infected with Avian Influenza Viruses. PLoS One. 2016; 11: e0153671.

41. Sutejo R, Yeo DS, Myaing MZ, Hui C, Xia J, Ko D, et al. Activation of type I and III interferon signalling pathways occurs in lung epithelial cells infected with low pathogenic avian influenza viruses. PLoS One. 2012; 7: e33732.

42. Abdelmohsen K, Hutchison ER, Lee EK, Kuwano Y, Kim MM, Masuda K, et al. miR-375 inhibits differentiation of neurites by lowering HuD levels. Molecular and cellular biology. 2010; 30: 4197-210.

43. Bronicki LM, Jasmin BJ. Emerging complexity of the HuD/ELAVl4 gene; implications for neuronal development, function, and dysfunction. Rna. 2013; 19: 1019-37.

44. Li H, Shang H, Shu D, Zhang H, Ji J, Sun B, et al. gga-miR-375 plays a key role in tumorigenesis post subgroup J avian leukosis virus infection. PLoS One. 2014; 9: e90878.

45. Denk J, Boelmans K, Siegismund C, Lassner D, Arlt S, Jahn H. MicroRNA Profiling of CSF Reveals Potential Biomarkers to Detect Alzheimer`s Disease. PLoS One. 2015; 10: e0126423.

46. Zehentmayr F, Hauser-Kronberger C, Zellinger B, Hlubek F, Schuster C, Bodenhofer $\mathrm{U}$, et al. Hsa-miR-375 is a predictor of local control in early stage breast cancer. Clinical epigenetics. 2016; 8: 28.

47. Shi ZC, Chu XR, Wu YG, Wu JH, Lu CW, Lu RX, et al MicroRNA-375 functions as a tumor suppressor in osteosarcoma by targeting PIK3CA. Tumour biology : the journal of the International Society for Oncodevelopmental Biology and Medicine. 2015; 36: 8579-84.

48. McGinnes LW, Morrison TG. Newcastle disease virus-like particles: preparation, purification, quantification, and incorporation of foreign glycoproteins. Current protocols in microbiology. 2013; 30: Unit 182.

49. Krutzfeldt J, Stoffel M. MicroRNAs: a new class of regulatory genes affecting metabolism. Cell metabolism. 2006; 4: 9-12.

50. Lotvall J, Hill AF, Hochberg F, Buzas EI, Di Vizio D, Gardiner C, et al. Minimal experimental requirements for definition of extracellular vesicles and their functions: a position statement from the International Society for Extracellular Vesicles. Journal of extracellular vesicles. 2014; 3: 26913.

51. Tkach M, Thery C. Communication by Extracellular Vesicles: Where We Are and Where We Need to Go. Cell. 2016; 164: 1226-32.

52. Pegtel DM, Cosmopoulos K, Thorley-Lawson DA, van Eijndhoven MA, Hopmans ES, Lindenberg JL, et al. Functional delivery of viral miRNAs via exosomes. Proceedings of the National Academy of Sciences of the United States of America. 2010; 107: 6328-33.

53. Bayraktar R, Van Roosbroeck K, Calin GA. Cell-to-cell communication: microRNAs as hormones. Molecular oncology. 2017; 11: 1673-86.

54. Pichlmair A, Reis e Sousa C. Innate recognition of viruses. Immunity. 2007; 27: 370-83.

55. Schoggins JW, Rice CM. Interferon-stimulated genes and their antiviral effector functions. Current opinion in virology. 2011; 1: 519-25.

56. Sen GC. Novel functions of interferon-induced proteins. Seminars in cancer biology. 2000; 10: 93-101.

57. Peteranderl C, Morales-Nebreda L, Selvakumar B, Lecuona E, Vadasz I, Morty $\mathrm{RE}$, et al. Macrophage-epithelial paracrine crosstalk inhibits lung edema clearance during influenza infection. J Clin Invest. 2016; 126: 1566-80.

58. Cai F, Zhu L, Wang F, Shi R, Xie XH, Hong X, et al. The Paracrine Effect of Degenerated Disc Cells on Healthy Human Nucleus Pulposus Cells Is Mediated by MAPK and NF-kappa B Pathways and Can Be Reduced by TGF-beta 1. DNA Cell Biol. 2017; 36: 143-58.

59. Noureddine MA, Qin XJ, Oliveira SA, Skelly TJ, van der Walt J, Hauser MA, et al. Association between the neuron-specific RNA-binding protein ELAVL4 and Parkinson disease. Hum Genet. 2005; 117: 27-33.

60. DeStefano AL, Latourelle J, Lew MF, Suchowersky O, Klein C, Golbe LI, et al. Replication of association between ELAVL4 and Parkinson disease: the GenePD study. Hum Genet. 2008; 124: 95-9.

61. Kim C, Lee H, Kang H, Shin JJ, Tak H, Kim W, et al. RNA-binding protein $\mathrm{HuD}$ reduces triglyceride production in pancreatic beta cells by enhancing the expression of insulin-induced gene 1. Biochimica et biophysica acta. 2016; 1859: $675-85$ 
62. Chen $\mathrm{CY}$, Shyu AB. HuD stimulates translation via eIF4A. Molecular cell. 2009; 36: 920-1.

63. Fukao A, Sasano Y, Imataka H, Inoue K, Sakamoto H, Sonenberg N, et al. The ELAV protein $\mathrm{HuD}$ stimulates cap-dependent translation in a Poly(A)- and eIF4A-dependent manner. Molecular cell. 2009; 36: 1007-17.

64. Tanner DC, Qiu S, Bolognani F, Partridge LD, Weeber EJ, Perrone-Bizzozero NI. Alterations in mossy fiber physiology and GAP-43 expression and function in transgenic mice overexpressing HuD. Hippocampus. 2008; 18: 814-23.

65. Choi KJ, Lee JH, Kim KS, Kang S, Lee YS, Bae S. Identification of ELAVL4 as a modulator of radiation sensitivity in A549 non-small cell lung cancer cells. Oncology reports. 2011; 26: 55-63.

66. Watanabe T, Aonuma H. Tissue-specific promoter usage and diverse splicing variants of found in neurons, an ancestral Hu/ELAV-like RNA-binding protein gene of insects, in the direct-developing insect Gryllus bimaculatus. Insect Mol Biol. 2014; 23: 26-41. 